\title{
WIPI2 depletion inhibits the growth of hepatocellular carcinoma cells through the AMPK signaling pathway
}

\author{
CHUNSHENG LIU ${ }^{1 *}$, FENG LI $^{2 *}$, XIAONING LI ${ }^{1}$, MINGGANG CAO $^{3}$, \\ GANG FENG $^{1}$, XIANWEN YUAN ${ }^{4,5}$ and XIAOLEI SHI ${ }^{4}$ \\ ${ }^{1}$ Department of Laboratory Medicine, Yijishan Hospital of Wannan Medical College, Wuhu, Anhui 241000; \\ ${ }^{2}$ Department of Gastroenterology, The First People's Hospital of Anqing, Anqing, Anhui 246000; \\ ${ }^{3}$ Department of Medicine, Anhui College of Traditional Chinese Medicine, Wuhu, Anhui 241000; \\ ${ }^{4}$ Department of Hepatobiliary Surgery, The Affiliated Drum Tower Hospital of Nanjing University Medical School, \\ Nanjing, Jiangsu 210008; ${ }^{5}$ State Key Laboratory of Pharmaceutical Biotechnology, Jiangsu Key Laboratory of \\ Molecular Medicine and School of Medicine, Nanjing University, Nanjing, Jiangsu 210093, P.R. China
}

Received November 4, 2019; Accepted February 12, 2020

DOI: 10.3892/or.2020.7531

\begin{abstract}
WD-repeat domain phosphoinositide-interacting protein 2 (WIPI2) is a protein that regulates the assembly of multiprotein complexes by presenting a beta-propeller platform for simultaneous and reversible protein-protein interactions. This study was designed to investigate the association between the expression of WIPI2 and the growth of hepatocellular carcinoma (HCC). Publicly-available data from the UALCAN platform revealed that WIPI2 is upregulated in tumor tissues compared with that noted in normal tissues in many types of tumors especially in HCC, and high WIPI2 expression predicts a poor patient prognosis. WIPI2 expression was significantly higher in tumor tissues compared with that in the corresponding adjacent normal tissues. Depletion of WIPI2 inhibited the proliferation and promoted the apoptosis
\end{abstract}

Correspondence to: Dr Xiaolei Shi or Dr Xianwen Yuan, Department of Hepatobiliary Surgery, The Affiliated Drum Tower Hospital of Nanjing University Medical School, 321 Zhongshan Road, Nanjing, Jiangsu 210008, P.R. China

E-mail: njsx12000@163.com

E-mail: yxw14612@163.com

${ }^{*}$ Contributed equally

Abbreviations: HCC, hepatocellular carcinoma; WIPI2, WD repeat domain phosphoinositide-interacting protein 2; Huh7/KD cells, Huh7 cells containing WIPI2 siRNA; Huh7/NC cells, Huh7 cells containing non-silencing sequence; Hep3B/KD cells, Hep3B cells containing WIPI2 siRNA; Hep3B/NC cells, Hep3B cells containing non-silencing sequence; $\mathrm{KD}$, knockdown; $\mathrm{NC}$, negative control; AMPK, adenosine 5'-monophosphate (AMP)-activated protein kinase

Key words: hepatocellular carcinoma, HCC, WIPI2, WD repeat domain phosphoinositide-interacting protein 2 , gene microarray both in HCC Huh7 and Hep3B cells. In order to explore the mechanisms of WIPI2 in HCC, WIPI2 was depleted in HCC cell lines and a gene microarray was constructed. The bioinformatic analysis showed that WIPI2 regulated the proliferation of HCC cells mainly through the AMPK signaling pathway. Further analysis indicated that the downstream factors of the AMPK signaling pathway were downregulated after WIPI2 depletion. Collectively, our study revealed that WIPI2 plays an important role in the pathogenesis of HCC mainly through the AMPK signaling pathway.

\section{Introduction}

Hepatocellular carcinoma (HCC) is one of the leading causes of cancer-related deaths worldwide and more than 600,000 deaths are attributed to HCC every year (1). In the last decade, many novel molecules targeting the HCC pathway have been discovered, exhibiting promising results (2). Although early detection and diagnosis play a critical role in reducing the incidence and mortality of HCC, the 5-year overall survival (OS) rate of HCC remains unsatisfactory (3). Previous research indicates that only $10-20 \%$ of HCC patients can undergo curative treatment at initial diagnosis, while the remaining patients are inoperable due to a poor prognosis $(4,5)$. Therefore, it is urgently necessary to explore new molecular targets for HCC treatment.

The family of WD-repeat proteins consists of more than 30 regulatory proteins within the beta-propeller fold. Originally, the WD-repeat has been identified as a core unit composed of approximately 40 amino acids, which ends with the residues, tryptophan and aspartic acid. The WD-repeat proteins regulate the assembly of multiprotein complexes by presenting a stable platform for simultaneous and reversible protein-protein interactions (6). By this means, WD-repeat proteins play important roles in many biological processes, including signal transduction, cell cycle control, apoptosis and chromatin assembly (7). The association between WD-repeat proteins and tumor phenotypes has been further studied by researchers (8). 
WD-repeat proteins are associated with the cell death pathway since previous studies have shown that mouse Apg16L acts as a scaffold in mammalian autophagy (9-11). WD-repeat domain phosphoinositide-interacting protein 2 (WIPI2) is reported to be regulated by mTORC1 to control autophagic flux (12).

In our previous study, we found that WIPI2 expression in HCC tissues was markedly increased when compared with that in adjacent normal cells, and WIPI was found to be associated with patient survival rate. Then we showed that the proliferation of cells was inhibited and the apoptosis rate was promoted after depletion of WIPI2 in HCC cell lines. Furthermore, we explored the molecular mechanisms underlying the WIPI2-mediated tumor cell growth using WIPI2-depleted and control cells. The results showed that the AMPK signaling pathway was significantly altered after WIPI 2 depletion. We also detected the downstream factors of the AMPK signaling pathway and found that the variation tendency was consistent with our hypothesis. Taken together, our findings support that WIPI2 regulates the growth of HCC cells mainly through the AMPK signaling pathway.

\section{Materials and methods}

Extraction and analysis of TCGA datasets. Data for WIPI2 expression and clinical information in the The Cancer Genome Atlas (TCGA) (https://www.cancer.gov/tcga) were extracted and analyzed in the UALCAN platform (http://ualcan.path. uab.edu) (13).

Tissue array and immunohistochemistry (IHC) assay. HCC tissue arrays were purchased from the National Engineering Center for Biochips (Shanghai, China). The expression of WIPI 2 in the tissues was evaluated by IHC assay using the WIPI2 antibody (dilution 1:150; cat. no. ab105459; Abcam). The tissue sections were collected, dewaxed in xylene, and rehydrated in graded ethanol solutions. All the following steps were carried out in a moist chamber. Then the tissue sections were blocked with $3 \%$ peroxide-methanol at room temperature for endogenous peroxidase ablation and incubated with blocking buffer (normal goat serum) at room temperature. After that, the blocking buffer was discarded and the sections were washed with PBS for 3 times. The sections were then incubated with purified primary antibody overnight at $4^{\circ} \mathrm{C}$, and incubated with the corresponding secondary antibody (dilution 1:200; cat. no. KGAA35; Nanjing KeyGen Biotech. Co. Ltd.). Finally, the sections were stained with DAB and hematoxylin. The staining was scored according to the staining intensity (no color, 0 ; faint yellow, 1; claybank, 2; brown, 3) and the proportion of positive cells $(0,<5 ; 1,<5$ and $<25 \% ; 2$, $<25$ and $<50 \%, 3,<50$ and $<75 \% ; 4,<75 \%$ positive cells), and the final staining scores were calculated as the score of the staining intensity multiplied by the score of the proportion of positive cells [score of 0 indicates negative staining ( $0+) ; 1-4$ is weak staining (1+); 5-8 is moderate staining (2+); 9-12 scores indicates strong staining $(3+)]$.

Cell culture. Huh7 and Hep3B cells were purchased from the Shanghai Institutes for Biological Sciences (China). The cells were maintained in DMEM (cat. no. 11965; Gibco; Thermo Fisher Scientific, Inc.) supplemented with $10 \%$ fetal bovine serum (FBS), $100 \mathrm{U} / \mathrm{ml}$ of penicillin and $100 \mu \mathrm{g} / \mathrm{ml}$ of streptomycin (complete medium) at $37^{\circ} \mathrm{C}$ in a humidified atmosphere containing $5 \% \mathrm{CO}_{2}$.

Small interfering RNA and transfection. The siRNAs targeting the WIPI2 sequence (KD, TACGGAAGATGTGTGCATT) and the non-silencing sequence (NC, TTCTCCGAACGTGTC ACGT) were purchased from Guangzhou RiboBio Co., Ltd. Transfection with siRNAs was completed using riboFECT CP transfection reagent (Guangzhou RiboBio Co., Ltd) according to the manufacturer's instructions.

MTT assay. Human cell lines ( $1 \times 10^{5}$ cells in $0.2 \mathrm{ml} /$ well) were seeded into 96-well plates and transfected with the siRNA and the non-silencing sequence. The cells were cultured at $37^{\circ} \mathrm{C}$ for $1-5$ days. Then $100 \mu 1(5 \mathrm{mg} / \mathrm{ml})$ of MTT solution was added into each well, followed by incubation at $37^{\circ} \mathrm{C}$ for $4 \mathrm{~h}$. Subsequently, the supernatant was removed, and $150 \mu \mathrm{l}$ of dimethyl sulfoxide (DMSO) was added into each well. The plate was oscillated at room temperature for $30 \mathrm{~min}$, and then the absorbance at a wavelength of $490 \mathrm{~nm}$ was determined by a multifunctional microplate reader (Tecan). The values were calculated after background subtraction. All the MTT experiments were repeated at least three times.

EdU incorporation assay. The cell proliferation was determined by the incorporation of 5-ethynyl-2'-deoxyuridine (EdU) into newly synthesized DNA strands, using a Click-iT EdU microplate assay kit (Beyotime Institute of Biotechnology) according to the manufacturer's instructions. The HCC cell lines were resuspended and seeded into a 6-well plate and transfected with the siRNA and negative control, respectively. Two hours before cell collection, EdU was added to the cells at a final concentration of $10 \mu \mathrm{M}$. After $2 \mathrm{~h}$, the incorporated EdU in DNA was coupled with Azide 488 dye, and subsequently the nuclei were stained with Hoechst 33342 . The cells were observed by fluorescence microscope at x100 magnification. The experiments were repeated at least three times.

TUNEL assay. Analysis of cell apoptosis was performed by terminal deoxynucleotidyl transferase-mediated dUTP nick-end labeling (TUNEL) assay (Nanjing KeyGen Biotech Co., Ltd.) according to the manufacturer's instructions. Following transfection for $24 \mathrm{~h}$, the cells were collected and fixed with $40 \mathrm{~g} / \mathrm{l}$ paraformaldehyde for $30 \mathrm{~min}$ at room temperature. The TdT enzyme solution was prepared and incubated with cells for $60 \mathrm{~min}$ at $37^{\circ} \mathrm{C}$. Next, the cells were washed with PBS and incubated with streptavidin-TRITC for $30 \mathrm{~min}$ at $37^{\circ} \mathrm{C}$. The cell nuclei were then stained with DAPI (dilution 1:1,000) at room temperature for $10 \mathrm{~min}$. Finally, the cells were mounted in anti-fade mounting medium and were observed with a fluorescence microscope at $\times 100$ magnification. The experiments were repeated at least three times.

Quantitative real-time PCR (RT-qPCR). Total RNA was extracted from HCC cell lines using TRIzol reagent (Invitrogen; Thermo Fisher Scientific, Inc.) following the manufacturer's instructions. RT-qPCR was performed as previously reported $(14,15)$. Briefly, $1 \mu \mathrm{g}$ of total RNA was reversely transcribed into cDNA using random primers and 
Table I. Primary and secondary antibodies used in the WB analysis.

\begin{tabular}{lccc}
\hline Antibody & Dilution & Catalog no. & Supplier \\
\hline WIPI2 & $1: 1,000$ & ab105459 & Abcam \\
GAPDH & $1: 1,000$ & 5174 & Cell Signaling Technology \\
$\beta$-actin & $1: 1,000$ & 3700 & Cell Signaling Technology \\
AMPK & $1: 1,000$ & 5831 & Cell Signaling Technology \\
p-AMPK & $1: 1,000$ & 50081 & Cell Signaling Technology \\
AKT & $1: 1,000$ & Cell Signaling Technology \\
p-AKT & $1: 1,000$ & Cell Signaling Technology \\
Caspase 3 & $1: 1,000$ & 5012 & Abcam \\
Bcl2 & $1: 1,000$ & 9662 & Abcam \\
Bax & $1: 1,000$ & ab32124 & Abcam \\
Cyclin D1 & $1: 8,000$ & Ab32503 & Abcam \\
Goat anti-mouse IgG H\&L (HRP) & $1: 10,000$ & ab134175 & Abcam \\
Goat anti-rabbit IgG H\&L (HRP) & $1: 10,000$ & ab205719 & ab205718
\end{tabular}

WB, western blot; WIPI2, WD-repeat domain phosphoinositide-interacting protein 2; GAPDH, glyceraldehyde-3-phosphate dehydrogenase; AMPK, AMP-activated protein kinase; AKT, protein kinase B; Bcl2, B-cell lymphoma 2; Bax, Bcl-2-associated X protein; p-, phosphorylated.

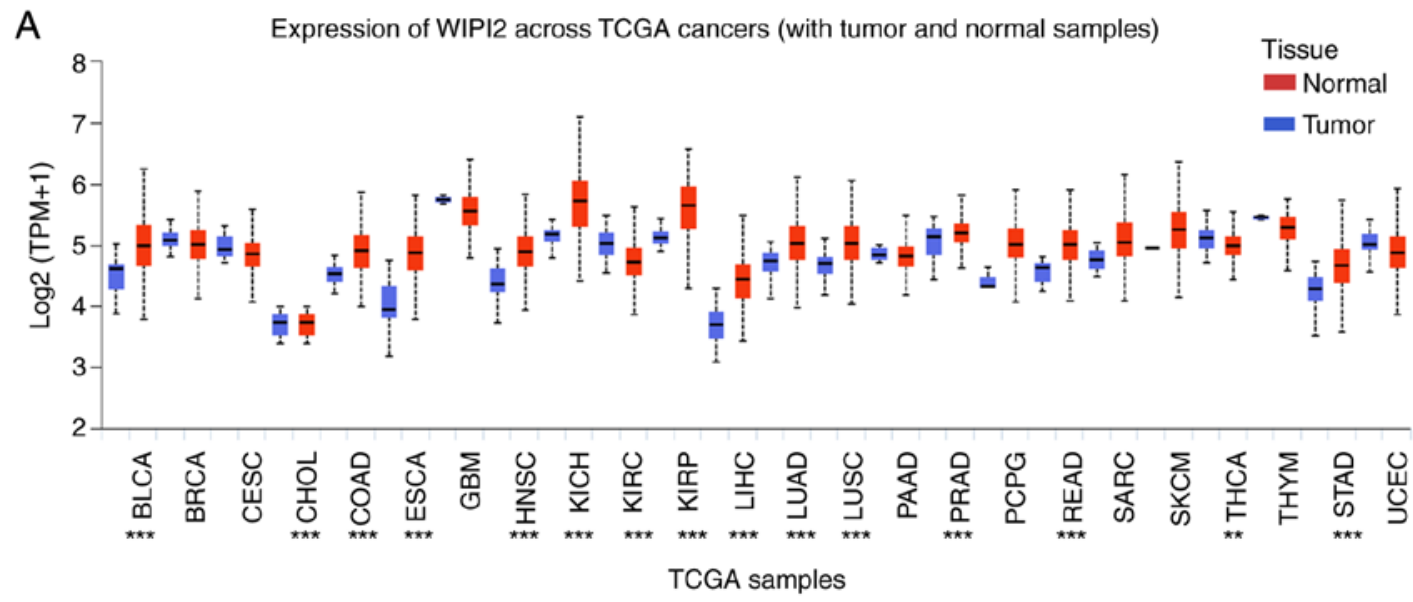

B Expression of WIPI2 in LIHC based on sample types

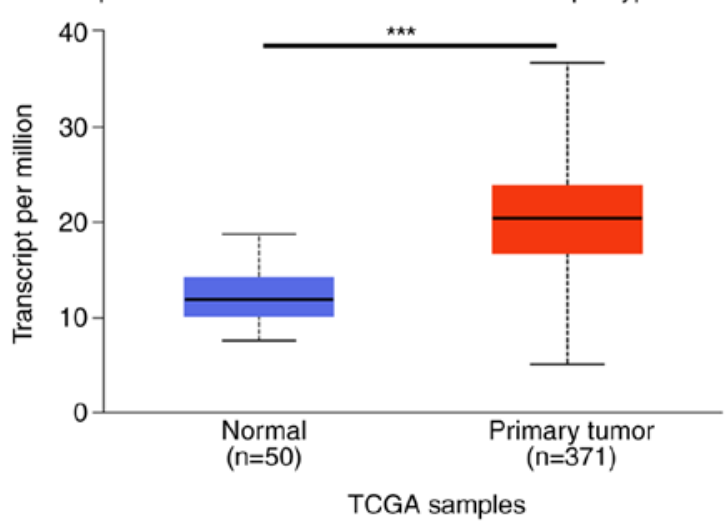

C Effect of WIPI2 expression $1.001 \quad$ level on LIHC patient survival

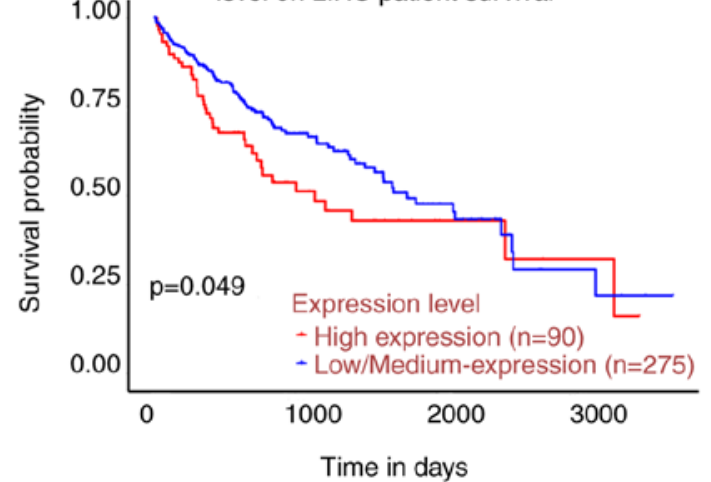

Figure 1. Expression analysis of WIPI2 based on the UALCAN platform. (A) Pan-cancer analysis of WIPI2 expression across cancers from the UALCAN platform. BLCA, bladder urothelial carcinoma; BRCA, breast invasive carcinoma; CESC, cervical squamous cell carcinoma and endocervical adenocarcinoma; CHOL, cholangiocarcinoma; COAD, colon adenocarcinoma; ESCA, esophageal carcinoma; GBM, glioblastoma multiforme; HNSC, head and neck squamous cell carcinoma; KICH, Kidney chromophobe; KIRC, kidney renal clear cell carcinoma; KIRP, kidney renal papillary cell carcinoma; LIHC, liver hepatocellular carcinoma; LUAD, lung adenocarcinoma; LUSC, lung squamous cell carcinoma; PAAD, pancreatic adenocarcinoma; PRAD, prostate adenocarcinoma; PCPG, pheochromocytoma and paraganglioma; READ, rectum adenocarcinoma; SARC, sarcoma; SKCM, skin cutaneous melanoma; THCA, thyroid carcinoma; THYM, thymoma; STAD, stomach adenocarcinoma; UCEC, uterine corpus endometrial carcinoma. ${ }^{* * *} \mathrm{P}<0.01,{ }^{* * *} \mathrm{P}<0.001$. (B) The WIPI2 level was higher in tumor tissues compared with that in normal tissues. ${ }^{* * *} \mathrm{P}<0.001$. (C) Survival probability revealed that high WIPI2 expression predicted poor prognosis, $\mathrm{P}<0.05$. WIPI2, WD repeat domain phosphoinositide-interacting protein 2. 
A
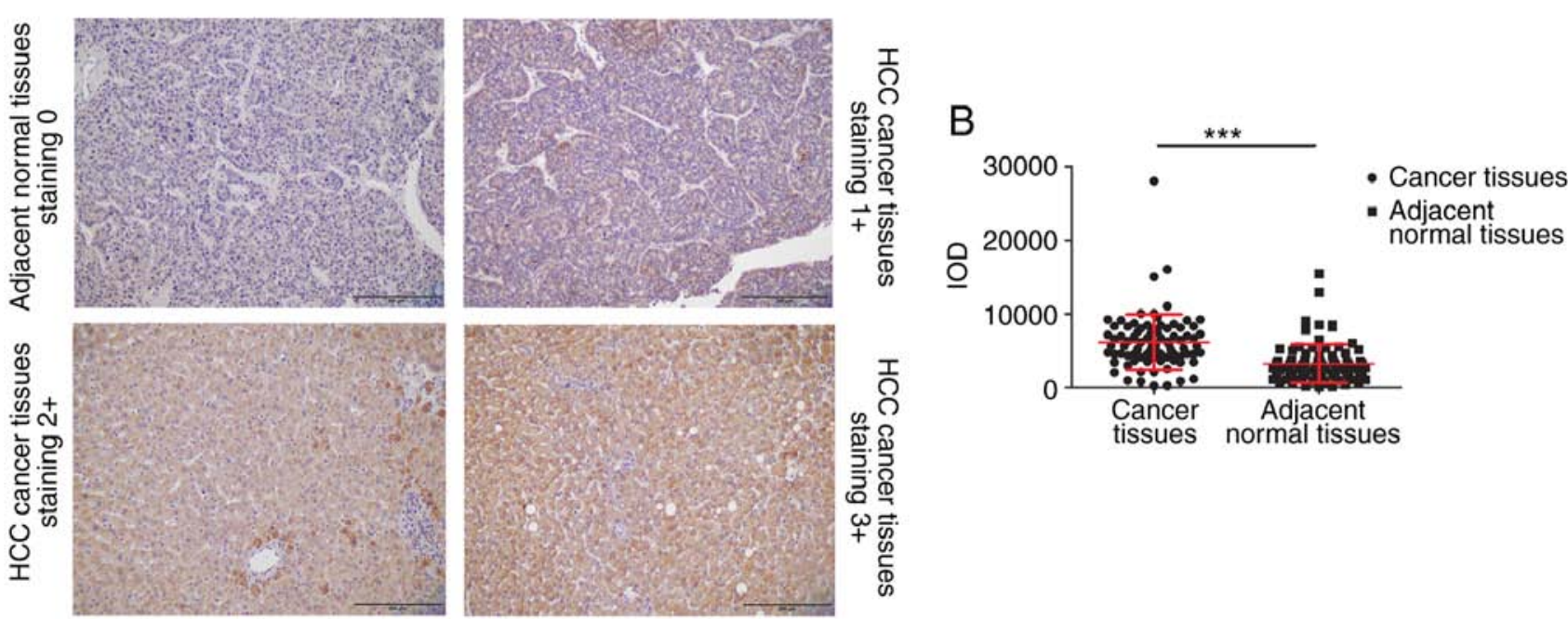

Figure 2. Immunohistochemical analysis of WIPI2 expression in human tissue microarrays. (A) Representative staining of WIPI2 expression in cancer tissues and corresponding adjacent normal tissues. Scale bar, $200 \mu \mathrm{m}$. (B) Integrated optical density (IOD) statistical analysis of WIPI2 in cancer tissues and corresponding adjacent normal tissues. ${ }^{* * * *} \mathrm{P}<0.001$. WIPI2, WD repeat domain phosphoinositide-interacting protein $2 ; \mathrm{HCC}$, hepatocellular carcinoma.

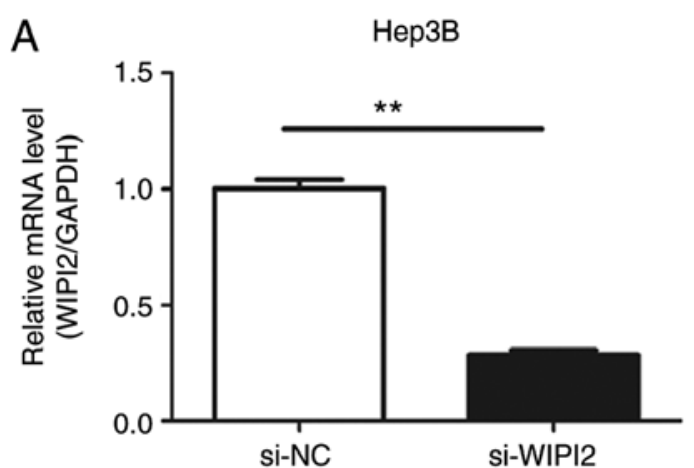

C


B

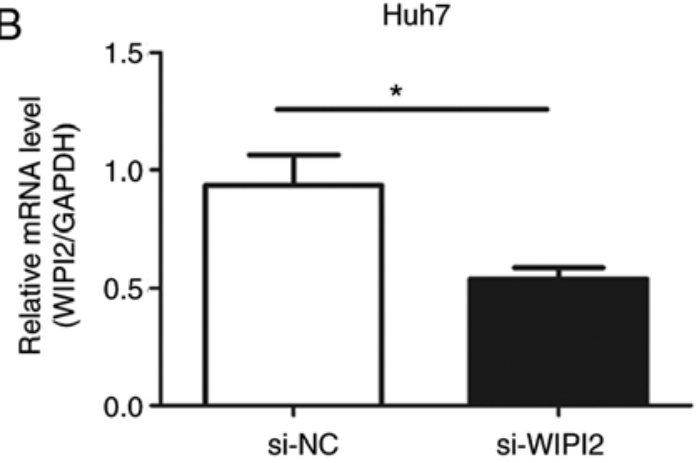

D

Huh7

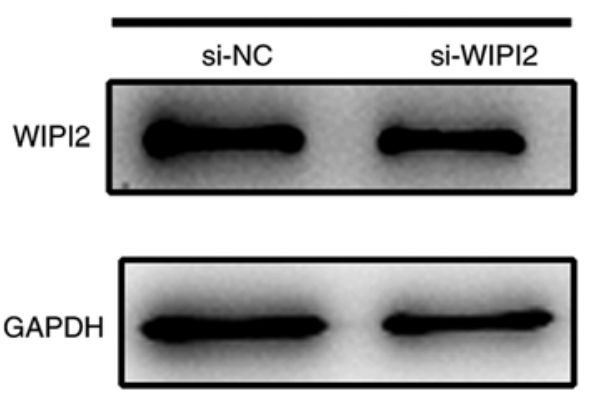

Huh7

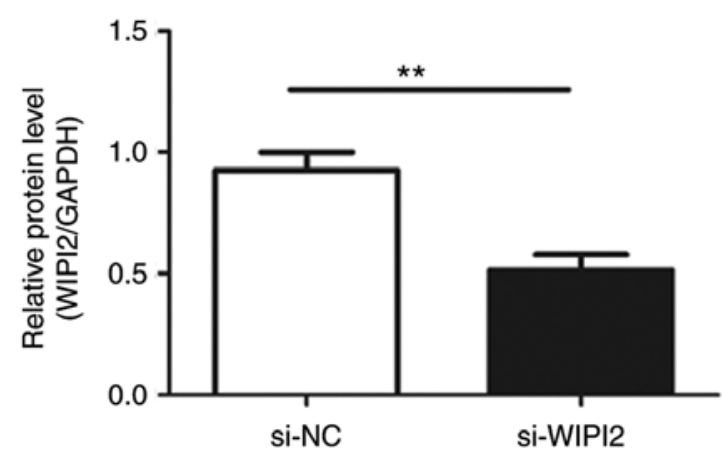

Figure 3. Efficiency of the silencing of WIPI2 in HCC cells. Expression of WIPI2 mRNA in (A) Hep3B and (B) Huh7 cells was examined by real-time PCR. Expression of WIPI2 protein in (C) Hep3B and (D) Huh7 cells was examined by western blot analysis. ${ }^{*} \mathrm{P}<0.05,{ }^{*} \mathrm{P}<0.01$. WIPI2, WD repeat domain phosphoinositide-interacting protein 2; HCC, hepatocellular carcinoma; si-WIPI2, WIPI2-knockdown group; si-NC, negative control group. 
Table II. Correlation of WIPI2 expression and clinicopathological features in the HCC tissue array.

\begin{tabular}{|c|c|c|c|c|c|c|c|c|c|}
\hline \multirow[b]{2}{*}{ Characteristics } & \multirow[b]{2}{*}{$\mathrm{N}$} & \multicolumn{5}{|c|}{ WIPI2 expression } & \multirow[b]{2}{*}{ Mean rank } & \multirow[b]{2}{*}{$\mathrm{Z}$} & \multirow[b]{2}{*}{ P-value } \\
\hline & & 0 & 1 & 2 & 3 & None & & & \\
\hline \multicolumn{10}{|l|}{ Sex } \\
\hline Male & 65 & 1 & 12 & 23 & 27 & 2 & 39.82 & -1.904 & 0.057 \\
\hline Female & 21 & 1 & 2 & 3 & 15 & 0 & 50.55 & & \\
\hline \multicolumn{10}{|l|}{ Age (years) } \\
\hline$<60$ & 28 & 2 & 12 & 7 & 6 & 1 & 32.02 & -2.989 & 0.003 \\
\hline$\geq 60$ & 58 & 3 & 15 & 1 & 38 & 1 & 47.46 & & \\
\hline \multicolumn{10}{|l|}{ Tumor size $(\mathrm{cm})$} \\
\hline$<5$ & 43 & 2 & 9 & 3 & 27 & 2 & 42.39 & -0.048 & 0.962 \\
\hline$\geq 5$ & 43 & 1 & 10 & 4 & 28 & 0 & 42.60 & & \\
\hline \multicolumn{10}{|l|}{ Differentiation } \\
\hline 1 & 7 & 1 & 3 & 1 & 2 & 0 & 31.07 & -0.608 & 0.543 \\
\hline 2 & 24 & 3 & 2 & 7 & 11 & 1 & 25.24 & & \\
\hline 3 & 52 & 1 & 21 & 0 & 29 & 1 & 51.74 & & \\
\hline 4 & 3 & 0 & 0 & 2 & 1 & 0 & 44.50 & & \\
\hline \multicolumn{10}{|l|}{ TNM stage } \\
\hline I & 17 & 0 & 2 & 1 & 13 & 1 & 14.25 & -0.581 & 0.561 \\
\hline II & 28 & 2 & 13 & 1 & 12 & 0 & 30.32 & & \\
\hline III & 40 & 1 & 4 & 14 & 20 & 1 & 63.00 & & \\
\hline IV & 1 & 0 & 0 & 1 & 0 & 0 & 36.00 & & \\
\hline \multicolumn{10}{|l|}{ Location } \\
\hline Tumor tissue & 86 & 1 & 17 & 10 & 56 & 2 & 109.79 & -6.800 & 0.000 \\
\hline Adjacent tissue & 86 & 44 & 13 & 6 & 23 & 0 & 61.77 & & \\
\hline
\end{tabular}

A tissue microarray was stained with the anti-human WIPI2 antibody (dilution 1:150, cat. no., ab105459, Abcam). The staining intensity was scored on a scale of five as follows: Negative $(0+)$, weak $(1+)$, moderate $(2+)$ and strong $(3+)$. None means that the site was lost in the section. $\mathrm{P}$-value denoted in bold indicates a significant difference. HCC, hepatocellular carcinoma; WIPI2, WD repeat domain phosphoinositide-interacting protein 2 .

Primescript reverse transcriptase (Takara). RT-qPCR for the indicated genes was carried out using the SYBR Green qPCR kit (Takara Dalian) on a fluorescent temperature cycler (Applied Biosystems ABI ${ }^{\mathrm{TM}}$ Vii7 Real Time PCR System; Thermo Fisher Scientific, Inc.). The following primers were used to detect the expression of WIPI2 (forward, 5'-CCATCG TCAGCCTTAAAGCAC-3' and reverse, 5'-TCCAGGCAT ACTATCAGCCTC-3') and GAPDH (forward, 5'-TGACTT CAACAGCGACACCCA-3' and reverse, 5'-CACCCTGTT GCTGTAGCCAAA-3'). Briefly, after an initial denaturation step at $95^{\circ} \mathrm{C}$ for $5 \mathrm{~min}$, amplifications were carried out with 45 cycles at a melting temperature of $95^{\circ} \mathrm{C}$ for $15 \mathrm{sec}$ and an annealing temperature of $60^{\circ} \mathrm{C}$ for $1 \mathrm{~min} . G A P D H$ was selected as an endogenous control, and the relative gene expression was determined by the comparative $\mathrm{Ct}$ method. The experiment was repeated at least three times.

Flow cytometry $(F C M)$. Cells transfected with the siRNA and control were harvested, and washed twice with cold PBS. Then the cells were stained with Annexin V-FITC and PI (eBioscience Inc.) and incubated for $15 \mathrm{~min}$ at room temperature, and then determined using a BD FACSCalibur Flow Cytometer
(BD Biosciences) and analyzed using FlowJo software (v10.0; TreeStar). The experiment was repeated at least three times.

Western blot analysis. Cells transfected with siRNA were harvested and total proteins were extracted from tumor cell lines using RIPA buffer containing fresh protease and phosphatase inhibitors. The protein concentration was determined using the BCA assay (Pierce; Thermo Fisher Scientific, Inc.). Briefly, equal amounts of proteins $(50 \mu \mathrm{g})$ were subjected to $10 \%$ SDS-PAGE and transferred onto PVDF membranes. The membranes were blocked with $3 \%$ BSA in $10 \mathrm{mM}$ Tris- $\mathrm{HCl}$ (pH 7.4) containing $0.05 \%$ Tween-20 and incubated with a primary antibody at $4^{\circ} \mathrm{C}$ for $12 \mathrm{~h}$. After washing with Tris- $\mathrm{HCl}$ buffer for three times, the membranes were incubated with a corresponding peroxidase-conjugated secondary antibody (Abcam). Immunoreactive bands were visualized using Super-Signal West Pico Chemiluminescent Substrate (Pierce; Thermo Fisher Scientific, Inc.). The densitometry of the protein bands was quantified by ImageJ software (1.8.0; National Institutes of Health). The experiment was repeated at least three times. The details of the primary antibodies used in the experiments are documented in Table I. 



Figure 4. WIPI2 knockdown suppresses the proliferation of HCC cells. (A) MTT assay was used to assess the cell proliferation in the HCC (A) Hep3B and (B) Huh7 cells following transfection with si-WIPI2 or si-NC. ${ }^{*} \mathrm{P}<0.05,{ }^{* *} \mathrm{P}<0.01$. EdU assays were conducted after WIPI2 depletion in (C) Hep3B and (D) Huh7 cells. Scale bar, $100 \mu \mathrm{m}$. (E) Statistical analysis of the EdU assay in (E) Hep3B and (F) Huh7 cells. * P<0.01. WIPI2, WD repeat domain phosphoinositide-interacting protein 2; HCC, hepatocellular carcinoma; si-WIPI2 or KD, WIPI2-knockdown group; si-NC or NC, negative control group.

Microarray and analysis. Total RNA was isolated from Hep3B cells transfected with si-WIPI2 ( $\mathrm{n}=3)$ and Hep3B cells transfected with si-NC $(\mathrm{n}=3)$. RNA samples were analyzed by microarray expression profiling using the Affymetrix Human GeneChip PrimeView (Affymetrix) according to the manufacturer's instructions. Briefly, cDNA target preparation and in vitro transcription were conducted using the GeneChip 3' IVT PLUS Kit (Affymetrix). Arrays were washed, stained and processed using the GeneChip Hybridization Wash and Stain Kit (Affymetrix), after which they were imaged using the Affymetrix GeneChip Scanner 3000 (Affymetrix) for subsequent generation of raw data. Genes significantly differentially expressed between the Hep3B/KD and Hep3B/NC cells were selected based on a threshold setting of fold change $>1.3$ and $\mathrm{P}<0.05$. Functional pathway analysis was conducted using Kyoto Encyclopedia of Genes and Genomes (KEGG) pathway enrichment analysis (16) and Gene Ontology (GO) analysis (17) according to the manufacturer's instructions.

Statistical analysis. Data are presented as the mean \pm standard deviation (SD) of three independent experiments. All statistical analyses were performed using SPSS 18.0 software (SPSS Inc.). $\mathrm{P}<0.05$ was considered statistically significant; $\mathrm{P}<0.01$ was considered statistically very significant. Differences among categorical variables were analyzed using one-way ANOVA/SNK test or independent-sample Student's t-test. The immunoreactive scores for WIPI2 for tissue array were analyzed using non-parametric Mann-Whitney U, Kruskal-Wallis $\mathrm{H}$ and Wilcoxon tests.

\section{Results}

Expression of WIPI2 is upregulated in HCC tumor tissues and WIPI2 predicts a poor prognosis. In all 24 types of cancers included in the UACLAN platform, WIPI2 expression was higher in tumor tissues compared with normal tissues (Fig. 1A and B). The survival rate showed that high WIPI2 expression predicted a poor patient prognosis (Fig. 1C).

WIPI2 expression was then detected in HCC tissue array by IHC assay. The array consisted of 86 HCC tissue pairs, including tumor and corresponding adjacent normal tissues. Among these samples, 65 tissue samples were obtained from 
A

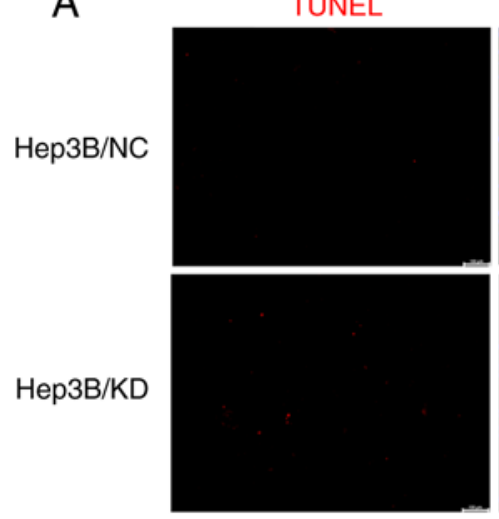

B

Huh7/NC

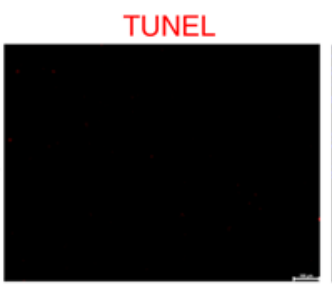

Huh7/KD

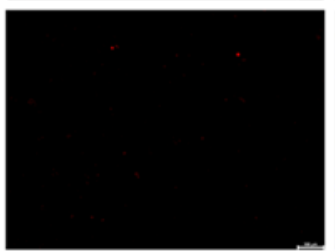

E



DAP



DAPI
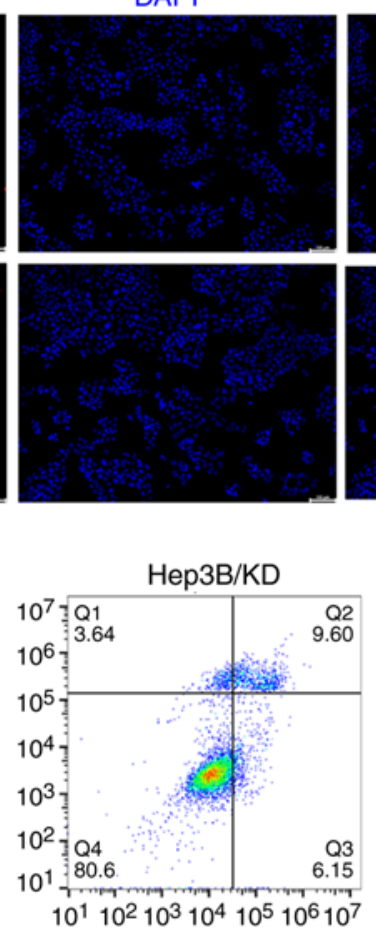

Merged

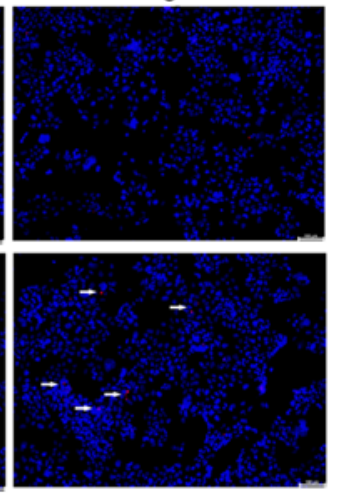

Merged
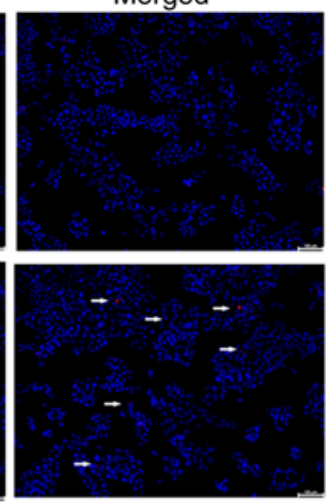

C

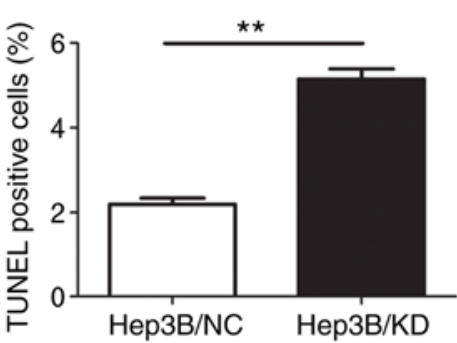

D

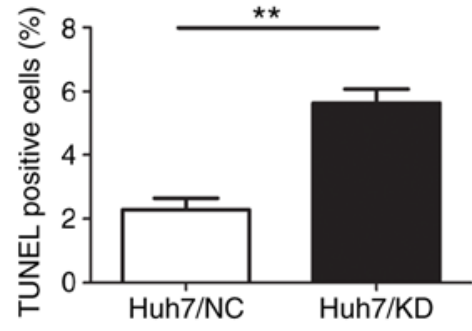

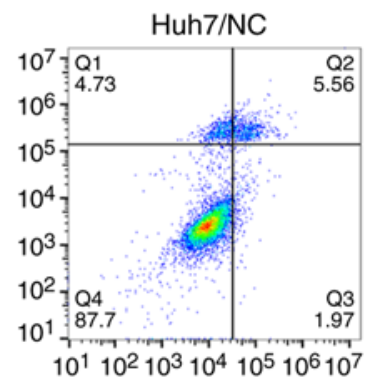



Annexin V-FITC
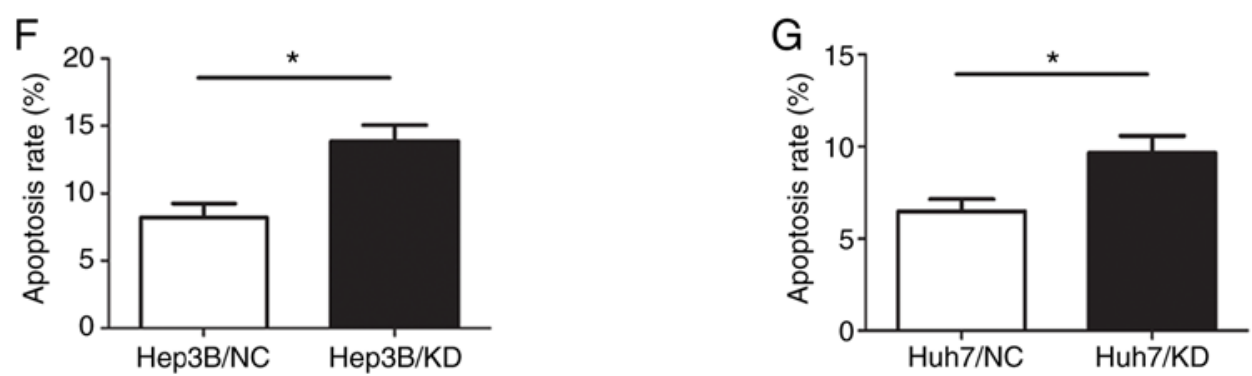

Figure 5. WIPI2 knockdown enhances the apoptosis of HCC cells. (A) TUNEL assay demonstrated that apoptosis was significantly enhanced in the (A) Hep3B and (B) Huh7 cell lines after WIPI2 knockdown. Arrows indicate TUNEL-positive cells. Scale bar, $100 \mu \mathrm{m}$. Statistical analyses of the TUNEL positive rate in the (C) Hep3B and (D) Huh7 cells. ${ }^{* *} \mathrm{P}<0.01$. (E) Flow cytometry (FCM) analyses indicated that the cell apoptosis rate in the Hep3B and Huh7 cells was significantly enhanced after WIPI2 knockdown. FCM statistical analysis in (F) Hep3B and (G) Huh7 cells. "P<0.05. WIPI2, WD repeat domain phosphoinositide-interacting protein 2; HCC, hepatocellular carcinoma; KD, WIPI2-knockdown group; NC, negative control group.

male patients, while 21 samples were collected from female patients, with a median age of 67 years (range, 26-88 years). Table II and Fig. 2A show that WIPI2 was highly enriched in HCC tumor tissue samples, evidenced by the fact that 66 tumor tissue samples showed a score of $\geq 2$. However, adjacent normal tissue samples displayed significantly reduced WIPI2 expression compared with tumor tissues $(\mathrm{P}<0.001)$. The results also indicated that WIPI 2 expression was markedly elevated in patients older than 60 years (Table II). In addition, WIPI2 expression was not associated with other factors. However, 


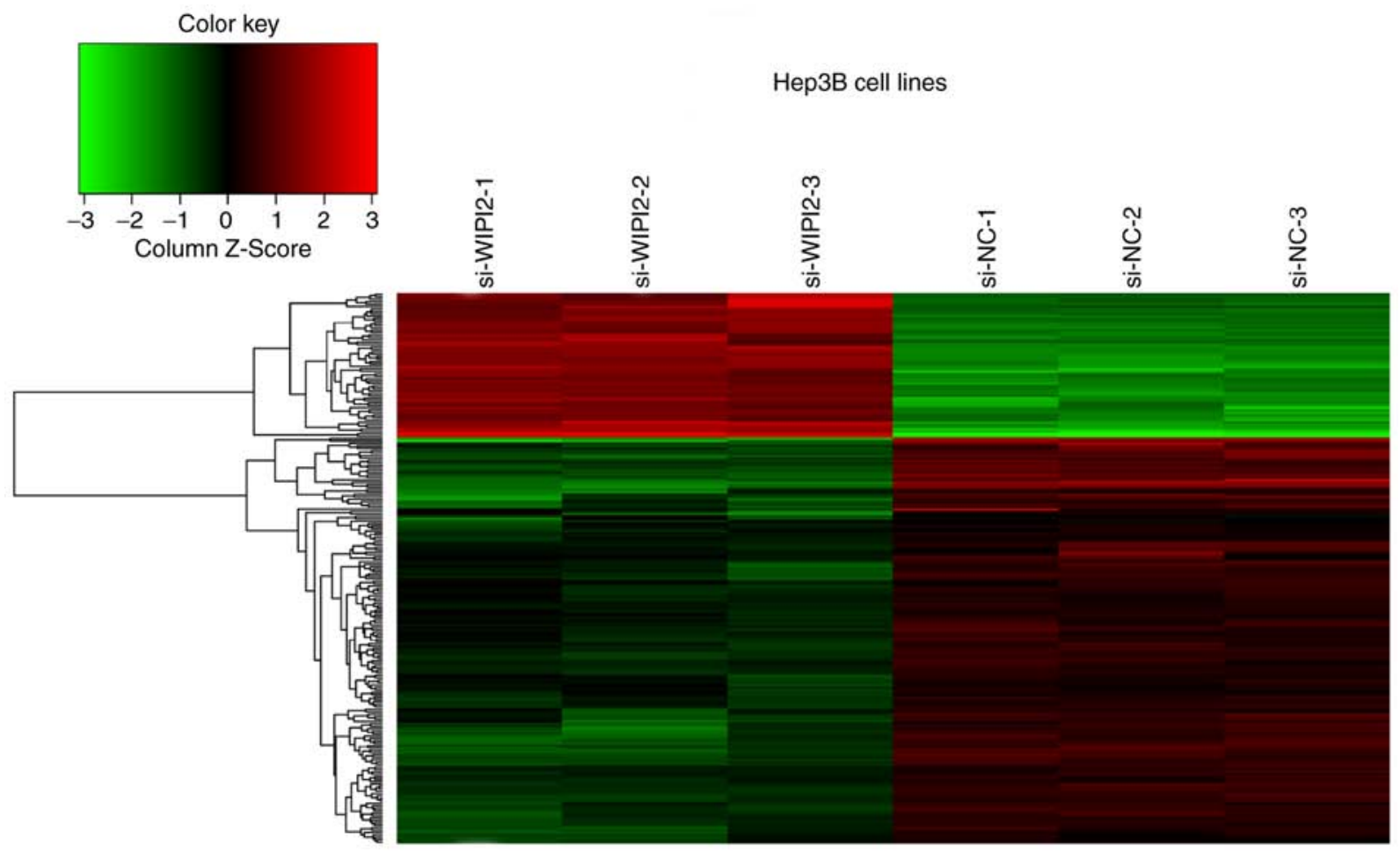

Figure 6. Heatmap of the expression levels of 262 dysregulated proteins. Gene microarray heatmap of Hep3B cells after WIPI2 downregulation. The red-colored clusters represent upregulated proteins, and the green-colored clusters represent downregulated proteins. WIPI2, WD repeat domain phosphoinositide-interacting protein 2; si-WIPI2, WIPI2-knockdown group; si-NC, negative control group.

the statistical analysis indicated that WIPI2 was significantly higher in tumor tissues than that in the corresponding adjacent normal tissues $(\mathrm{P}<0.001)$ (Fig. 2B).

Downregulation of WIPI2 suppresses the proliferation and increases the cytotoxicity of HCC cells. In the present study, siRNA targeting WIPI2 was employed to suppress WIPI2 expression in the HCC cell lines in order to verify its roles in the tumorigenesis of HCC in vitro. After transfection with siRNA, the expression of WIPI2 at the mRNA and protein levels were both decreased in the HCC Huh7 and Hep3B cell lines (Fig. 3A-D).

In order to determine the effect of WIPI2 on the cell proliferation of HCC cells, MTT and EdU assays were used after WIPI2 downregulation. MTT results showed that the proliferation of HCC cells was increased following WIPI2 depletion (si-WIPI2) (Fig. 4A and B). In addition, the EdU assay indicated that cell proliferation was significantly decreased after WIPI2 ablation. The number of EdU-positive cells was significantly reduced upon WIPI2 knockdown (KD) in contrast to the negative control (NC) cells (Fig. 4C-F).

Knockdown of WIPI2 increases the apoptosis of HCC cells. To explore the role of WIPI2 in regulating the growth of HCC cells, we assessed the changes in apoptosis after WIPI2 depletion by TUNEL assay and FCM. The results of TUNEL staining revealed that the percentage of TUNEL-positive cells was significantly increased after WIPI2 knockdown (KD) compared with the negative control (NC) cells (Fig. 5A-D). Consistently, the FCM results indicated that the apoptotic rate of HCC cells was significantly increased after
WIPI2 knockdown both in the Huh7 and Hep3B cell lines (Fig. 5E and F). These results showed that WIPI2 ablation could promote the apoptosis of HCC cells.

Depletion of WIPI2 inhibits cell proliferation through the $A M P K$ signaling pathway. To uncover the mechanism underlying the WIPI2-induced proliferation and apoptosis, we conducted a microarray analysis to identify differentially expressed genes upon WIPI2 depletion using Hep3B/KD and Hep3B/NC cell lines (Fig. 6). The results showed that a total of 63 genes were upregulated, and 199 genes were downregulated after WIPI 2 depletion $(\mathrm{P}<0.05$ and fold change $>1.3$ ), and all the details of the 216 gene symbols and the fold changes are shown in Table SI. Supervised analysis was conducted following the KEGG and GO technical route (Fig. 7A and B). We found that the AMPK signaling pathway was markedly modified after WIPI2 depletion.

In regards to the downstream factors of the AMPK pathway, AKT is associated with cell proliferation and apoptosis. Thus, we hypothesized that AKT may be involved in the inhibition of HCC cells. Further verification was conducted by western blot analysis. Phosphorylation of AKT (p-AKT) was significantly decreased, while total AKT remained unchanged after WIPI depletion (Fig. 8A and C) in the Hep3B cells. The results also revealed that the ratios of $\mathrm{p}-\mathrm{AMPK} / \mathrm{AMPK}$ and $\mathrm{p}-\mathrm{AKT} / \mathrm{AKT}$ were reduced after WIPI2 knockdown (Fig. 8E). Cyclin D1 is associated with cell proliferation; thus, we detected the expression of cyclin D1 and found that it was significantly decreased after WIPI2 depletion (Fig 8A and C) in the Hep3B cells. Since TUNEL assay indicated that the apoptosis was increased, we also determined the levels of Bcl2, Bax, 
A

$\square$ Positive z-score $\square$-socre $=0$ Negative z-score $\square$ No activity pattern available $\quad$ -
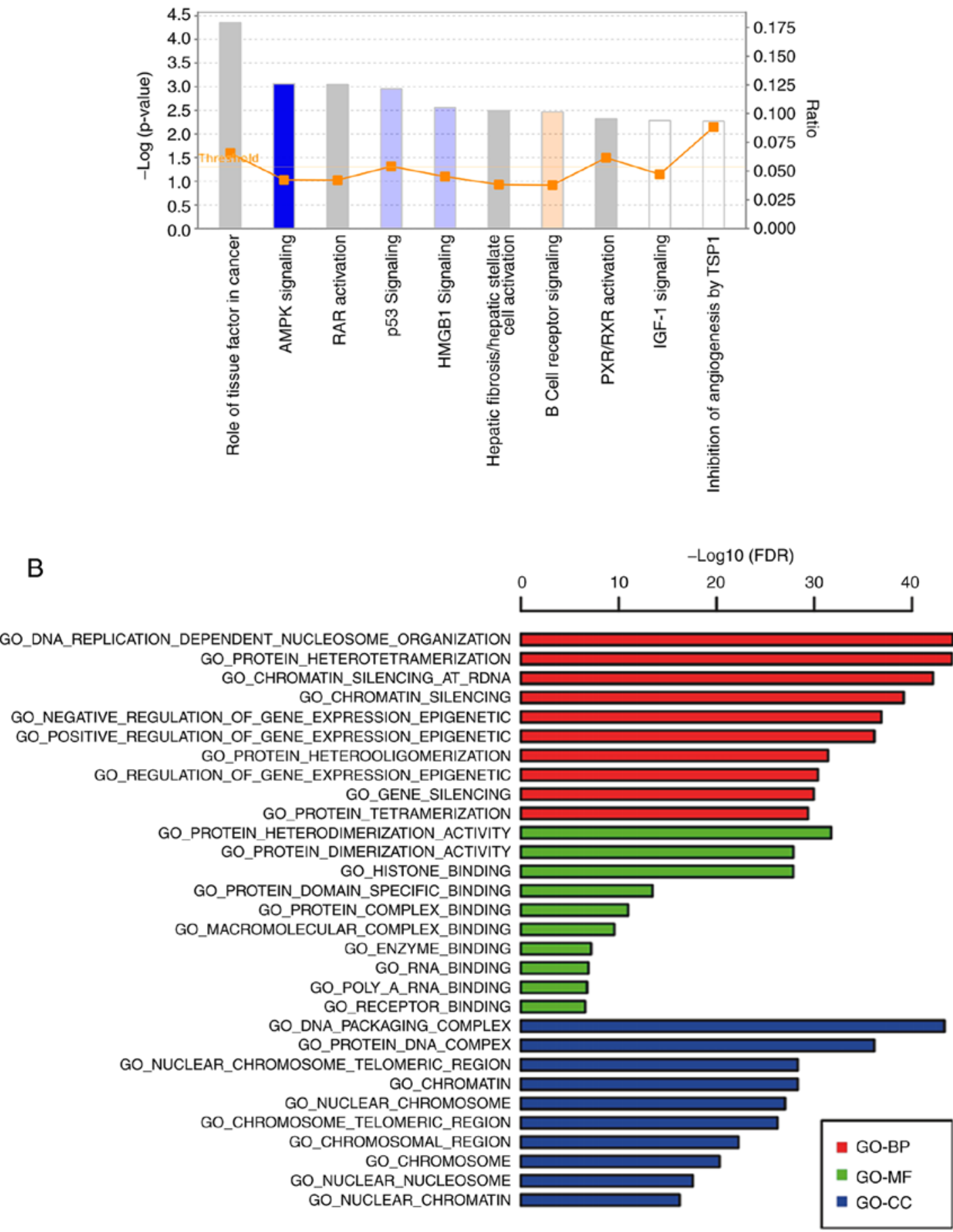

Figure 7. Canonical pathway and Gene Ontology (GO) enrichment analysis of gene microarray. (A) Distribution of enriched canonical pathway analysis indicates that the AMPK pathway is significantly downregulated after WIPI2 knockdown. (B) An overview of the GO annotations of the 262 dysregulated proteins in three categories: Biological process (GO-BP), molecular function (GO-MF) and cellular component (GO-CC). WIPI2, WD repeat domain phosphoinositide-interacting protein 2; AMPK, adenosine 5'-monophosphate (AMP)-activated protein kinase.

caspase-3 and cleaved-caspase 3, key biomarkers of apoptosis (Fig. 8B and D). The results indicated that the $\mathrm{Bcl} 2 / \mathrm{Bax}$ ratio was decreased (Fig. 8F) and cleaved-caspase 3 was also evaluated and caspase-3 was not altered (Fig. 8B and D) after WIPI2 knockdown. Similar results were observed in the Huh7 cells (Fig. 8G-L). Taken together, our data revealed that WIPI2 regulated the growth of HCC cells mainly through the AMPK pathway.

\section{Discussion}

In the present study, we first demonstrated the association between WD repeat domain phosphoinositide-interacting protein 2 (WIPI2) expression and prognosis of HCC patients. We found that WIPI2 expression was increased in human HCC tissues when compared with that in adjacent normal tissues. In vitro experiments showed that knockdown of WIPI2 



Figure 8. Mechanisms underlying the WIPI2-mediated regulation of the proliferation of HCC cells. (A) Determination of various downstream factors associated with cell proliferation of the AMPK pathway by western blot analysis in the Hep3B cells in the WIPI2-knockdown (KD) or negative control (NC) groups. (B) Detection of various apoptotic biomarkers by western blot analysis in the Hep3B cells in the two groups. (C) Statistical analysis of proteins in the Hep3B cells. ${ }^{*} \mathrm{P}<0.05$. (D) Statistical analysis of apoptosis biomarkers in the Hep3B cells. ${ }^{* *} \mathrm{P}<0.01$. (E) Phosphorylated protein/total protein ratio analysis after WIPI2 knockdown in the Hep3B cells. ${ }^{*} \mathrm{P}<0.05,{ }^{* *} \mathrm{P}<0.01$. (F) Bcl2/Bax ratio analysis after WIPI2 knockdown in the Hep3B cells. ${ }^{* *} \mathrm{P}<0.01$. (G) Determination of various downstream factors associated with cell proliferation of the AMPK pathway by western blot analysis in the Huh7 cells in the WIPI2-knockdown (KD) or negative control (NC) groups. (H) Detection of various apoptotic biomarkers by western blot analysis in the Huh7 cells in the two groups. (I) Statistical analysis of proteins in the Huh7 cells. ${ }^{*} \mathrm{P}<0.05,{ }^{* *} \mathrm{P}<0.01$. (J) Statistical analysis of apoptosis biomarker in the Huh7 cells. ${ }^{*} \mathrm{P}<0.05$, ${ }^{* *} \mathrm{P}<0.01$. (K) Phosphorylated protein/total protein ratio analysis after WIPI2 knockdown in the Huh7 cells, ${ }^{*} \mathrm{P}<0.05,{ }^{* * *} \mathrm{P}<0.01$. (L) Bcl2/Bax ratio analysis after WIPI2 knockdown in the Huh7 cells. ${ }^{* *} \mathrm{P}<0.01$. WIPI2, WD repeat domain phosphoinositide-interacting protein 2; AMPK, adenosine 5 '-monophosphate (AMP)-activated protein kinase; AKT, protein kinase B; Bcl2, B-cell lymphoma 2; Bax, Bcl-2-associated X protein.

significantly inhibited the proliferation and promoted the apoptosis of HCC cell lines.

In order to reveal how WIPI2 regulated the pathogenesis of HCC, we performed RNA microarray and western blot analysis. The bioinformatic analysis indicated that the AMPK signaling pathway is significantly modified after WIPI2 depletion. As a heterotrimer, AMPK consists of a catalytic subunit $(\alpha)$ and two regulatory subunits ( $\beta$ and $\gamma$ ) (18). Compound C [6-(4-(2-piperidin-1-ylethoxy) phenyl)-3-pyridin-4-ylpyrazolo(1,5-a)pyrimidine] is a selective AMPK inhibitor (19). Moreover, inhibition of AMPK induced by compound $\mathrm{C}$ can lead to cell cycle arrest and apoptosis (19). Glioma cell growth was found to be inhibited by treatment of compound $\mathrm{C}$, and the apoptotic effect was also observed after AMPK knockdown (20). Further research revealed that human glioma treated with inhibitor of AMPK could be suppressed by multiple mechanisms containing inhibition of AKT/mTOR, G2-M cell cycle arrest, 
and induction of apoptosis mediated with caspase 3 and $\mathrm{Bcl} 2$ (20). AMPK downregulation was able to reduce the proliferation of many solid cancer cell lines such as HeLa, PC12 and prostate cancer cells $(21,22)$. We hypothesize that the AMPK/AKT axis is important for cell proliferation and apoptosis. In the present study, we further detected the levels of AMPK, p-AMPK, AKT and p-AKT and found that the AMPK pathway was significantly inhibited after WIPI2 ablation. And these results were consistent with the above microarray assay.

The AKT pathway plays a vital role in many fundamental cellular progresses including proliferation, survival, apoptosis and metabolism $(23,24)$. AKT is activated by AMPK phosphorylation $(25,26)$. After activation, AKT translocates to the cytoplasm or nucleus to phosphorylate its substrates. Cyclin D1 is a key molecule that is involved in G1-S phase transition, and its expression is associated with cell proliferation (27). Cyclin D1 is an important downstream factor of the AKT pathway (28), thus we detected cyclin D1 expression. Western blot analysis indicated that cyclin D1 was significantly decreased accompanied by a decrease in p-AMPK and p-AKT after WIPI2 knockdown.

Apoptosis is a process of programmed cell death mainly through the caspase cascade and the $\mathrm{Bcl} 2$ gene family. The caspases consist of 'initiator' caspases such as caspase-6, capase-8, caspase-9 and 'effector' caspases including caspase-2, caspase-3 and caspase 7 (29). When apoptosis starts, caspase- 3 functions as an effector. The apoptosis induced by the $\mathrm{Bcl} 2$ gene family is determined by the ratio of $\mathrm{Bcl} 2 / \mathrm{Bax}$. A higher ratio of $\mathrm{Bcl} 2 / \mathrm{Bax}$ reduces cell apoptosis (30). In the present study, flow cytometry and TUNEL assay showed that knockdown of WIPI2 induced apoptosis, and AMPK inhibition also induced apoptosis (20). We further determined the level of $\mathrm{Bcl} 2$, Bax and cleaved-caspase-3, and found that apoptosis was increased after WIPI2 downregulation. Taken together, the study indicated that WIPI2 regulates the growth of HCC cells, at least partly, through the AMPK pathway.

Collectively, our present study identified that WIPI2 expression is increased in HCC tissues when compared with that in adjacent normal tissues and high WIPI2 expression predicts a poor patient prognosis. Moreover, WIPI2 modulates the growth of HCC cells mainly through the AMPK/AKT/cyclin D1 axis and induces apoptosis via caspase- 3 and $\mathrm{Bcl} 2$. Therefore, WIPI 2 may be employed as a potential therapeutic target for HCC.

\section{Acknowledgements}

Not applicable.

\section{Funding}

This study was supported by the National Natural Science Foundation of China (nos. 31300103 and 81670566), and the funding body aided us in the design of the study, collection and analysis of the data. This study was also supported by the Research of Institute of Hospital Management, Nanjing University (NDYG2017016), and the funding body aided in the interpretation of the data and writing of the manuscript.

\section{Availability of data and materials}

The datasets used and analyzed during the present study are available from the corresponding author on reasonable request.

\section{Authors' contribution}

XS and XY conceived and designed this study. CL, FL, MC, XL, GF and XY performed the experiments. XY and XS collected and analyzed the data. XS drafted the manuscript. All authors read and approved the manuscript and agree to be accountable for all aspects of the research in ensuring that the accuracy or integrity of any part of the work are appropriately investigated and resolved.

\section{Ethics approval and consent to participate}

The human samples used in the present study were purchased from Shanghai Outdo Biotech (Shanghai, China). The company is registered in the National Human Genetic Resources Sharing Service Platform. The web site is: http://www.egene. org.cn/cms/g-index.jhtml, and the registration number is 2005DKA21300. The samples of this array were obtained from Taizhou Hospital of Zhejiang Province, and the use of this array was approved by the Ethics Committee of Taizhou Hospital of Zhejiang Province, China. Written informed consent was obtained from the patients.

\section{Patient consent for publication}

Consent was obtained from all individual participants included in the study.

\section{Competing interests}

The authors state that they have no competing interests.

\section{References}

1. Baradaran Noveiry B, Hirbod-Mobarakeh A, Khalili N, Hourshad N, Greten TF, Abou-Alfa GK and Rezaei N: Specific immunotherapy in hepatocellular cancer: A systematic review. J Gastroenterol Hepatol 32: 339-351, 2017.

2. Ye S, Zhao XY, Hu XG, Li T, Xu QR, Yang HM, Huang DS and Yang L: TP53 and RET may serve as biomarkers of prognostic evaluation and targeted therapy in hepatocellular carcinoma. Oncol Rep 37: 2215-2226, 2017.

3. El-Serag HB: Hepatocellular carcinoma. N Engl J Med 365: 1118-1127, 2011.

4. Ferlay J, Shin HR, Bray F, Forman D, Mathers C and Parkin DM: Estimates of worldwide burden of cancer in 2008: GLOBOCAN 2008. Int J Cancer 127: 2893-2917, 2010.

5. Rampone B, Schiavone B and Confuorto G: Current management of hepatocellular cancer. Curr Oncol Rep 12: 186-192, 2010.

6. Grimmel M,Backhaus Cand Proikas-Cezanne T: WIPI-mediated autophagy and longevity. Cells 4: 202-217, 2015.

7. Yu TW, Mochida GH, Tischfield DJ, Sgaier SK, Flores-Sarnat L, Sergi CM, Topçu M, McDonald MT, Barry BJ, Felie JM, et al: Mutations in WDR62, encoding a centrosome-associated protein, cause microcephaly with simplified gyri and abnormal cortical architecture. Nat Genet 42: 1015-1020, 2010.

8. Casper AL, Baxter K and Van Doren M: No child left behind encodes a novel chromatin factor required for germline stem cell maintenance in males but not females. Development 138: 3357-3366, 2011. 
9. Dooley HC, Razi M, Polson HE, Girardin SE, Wilson MI and Tooze SA: WIPI2 links LC3 conjugation with PI3P, autophagosome formation, and pathogen clearance by recruiting Atg12-5-16L1. Mol Cell 55: 238-252, 2014.

10. Axe EL, Walker SA, Manifava M, Chandra P, Roderick HL, Habermann A, Griffiths G and Ktistakis NT: Autophagosome formation from membrane compartments enriched in phosphatidylinositol 3-phosphate and dynamically connected to the endoplasmic reticulum. J Cell Biol 182: 685-701, 2008.

11. Itakura $\mathrm{E}$ and Mizushima $\mathrm{N}$ : Characterization of autophagosome formation site by a hierarchical analysis of mammalian Atg proteins. Autophagy 6: 764-776, 2010.

12. Wan W, You Z, Zhou L, Xu Y, Peng C, Zhou T, Yi C, Shi Y and Liu W: mTORC1-regulated and HUWE1-mediated WIPI2 degradation controls autophagy flux. Mol Cell 72: 303-315.e6, 2018.

13. Chandrashekar DS, Bashel B, Balasubramanya SAH, Creighton CJ, Ponce-Rodriguez I, Chakravarthi BVSK and Varambally S: UALCAN: A portal for facilitating tumor subgroup gene expression and survival analyses. Neoplasia 19: 649-658, 2017

14. Yuan X, Sun X, Shi X, Jiang C, Yu D, Zhang W and Ding Y: USP39 regulates the growth of SMMC-7721 cells via FoxM1. Exp Ther Med 13: 1506-1513, 2017.

15. Yuan X, Sun X, Shi X, Jiang C, Yu D, Zhang W, Guan W, Zhou J, Wu Y, Qiu Y and Ding Y: USP39 promotes the growth of human hepatocellular carcinoma in vitro and in vivo. Oncol Rep 34: 823-832, 2015.

16. Kanehisa M, Sato Y, Furumichi M, Morishima K and Tanabe M: New approach for understanding genome variations in KEGG. Nucleic Acids Res 47 (D1): D590-D595, 2019.

17. The Gene Ontology Consortium: The gene ontology resource: 20 years and still GOing strong. Nucleic Acids Res 47 (D1): D330-D338, 2019.

18. Hardie DG and Alessi DR: LKB1 and AMPK and the cancer-metabolism link-ten years after. BMC Biol 11: 36, 2013.

19. Akimoto T, Umemura M, Nagasako A, Ohtake M, Fujita T, Yokoyama U, Eguchi $\mathrm{H}$, Yamamoto $\mathrm{T}$ and Ishikawa $\mathrm{Y}$ : Alternating magnetic field enhances cytotoxicity of Compound C. Cancer Sci 109: 3483-3493, 2018.

20. Liu X, Chhipa RR, Nakano I and Dasgupta B: The AMPK inhibitor compound $\mathrm{C}$ is a potent AMPK-independent antiglioma agent. Mol Cancer Ther 13: 596-605, 2014.

21. Shaw MM, Gurr WK, McCrimmon RJ, Schorderet DF and Sherwin RS: 5'AMP-activated protein kinase alpha deficiency enhances stress-induced apoptosis in BHK and PC12 cells. J Cell Mol Med 11: 286-298, 2007.
22. Duong HQ, Hwang JS, Kim HJ, Seong YS and Bae I: BML-275, an AMPK inhibitor, induces DNA damage, G2/M arrest and apoptosis in human pancreatic cancer cells. Int $\mathrm{J}$ Oncol 41: 2227-2236, 2012

23. Chen Y, Wang H, Zhang Y, Wang Z, Liu S and Cui L: Pretreatment of ghrelin protects $\mathrm{H} 9 \mathrm{c} 2$ cells against hypoxia/reoxygenation-induced cell death via PI3K/AKT and AMPK pathways. Artif Cells Nanomed Biotechnol 47: 2179-2187, 2019.

24. Alam H, Weck J, Maizels E, Park Y, Lee EJ, Ashcroft M and Hunzicker-Dunn M: Role of the phosphatidylinositol-3-kinase and extracellular regulated kinase pathways in the induction of hypoxia-inducible factor (HIF)-1 activity and the HIF-1 target vascular endothelial growth factor in ovarian granulosa cells in response to follicle-stimulating hormone. Endocrinology 150: 915-928, 2009.

25. Yang M, Zhang Z, Wang C, Li K, Li S, Boden G, Li L and Yang G: Nesfatin-1 action in the brain increases insulin sensitivity through Akt/AMPK/TORC2 pathway in diet-induced insulin resistance. Diabetes 61: 1959-1968, 2012.

26. Hur W, Lee JH, Kim SW, Kim JH, Bae SH, Kim M, Hwang D, Kim YS, Park T, Um SJ, et al: Downregulation of microRNA-451 in non-alcoholic steatohepatitis inhibits fatty acid-induced proinflammatory cytokine production through the AMPK/AKT pathway. Int J Biochem Cell Biol 64: 265-276, 2015.

27. Wei Y, Huang C, Wu H and Huang J: Estrogen receptor Beta $(\mathrm{ER} \beta)$ mediated-cyclin D1 degradation via autophagy plays an anti-proliferation role in colon cells. Int J Biol Sci 15: 942-952, 2019.

28. He YY, Council SE, Feng L and Chignell CF: UVA-induced cell cycle progression is mediated by a disintegrin and metalloprotease/epidermal growth factor receptor/AKT/Cyclin D1 pathways in keratinocytes. Cancer Res 68: 3752-3758, 2008.

29. Dubey M, Nagarkoti S, Awasthi D, Singh AK, Chandra T, Kumaravelu J, Barthwal MK and Dikshit M: Nitric oxide-mediated apoptosis of neutrophils through caspase- 8 and caspase-3-dependent mechanism. Cell Death Dis 7: e2348, 2016.

30. Maes ME, Schlamp CL and Nickells RW: BAX to basics: How the BCL2 gene family controls the death of retinal ganglion cells. Prog Retin Eye Res 57: 1-25, 2017.

This work is licensed under a Creative Commons Attribution-NonCommercial-NoDerivatives 4.0 International (CC BY-NC-ND 4.0) License. 